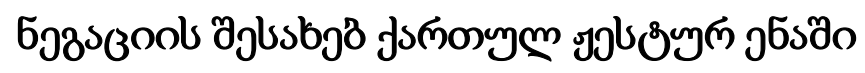

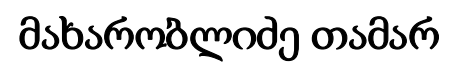

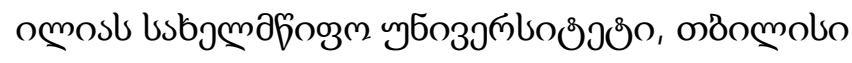

https://doi.org/10.52340/idw.2021.508

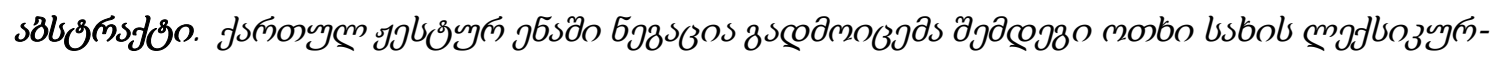

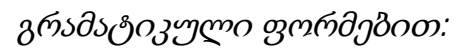

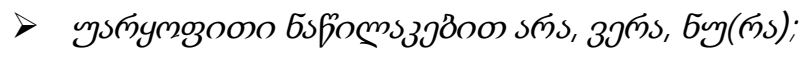

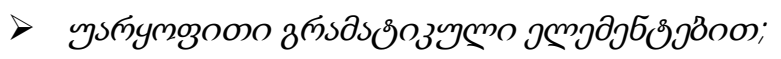

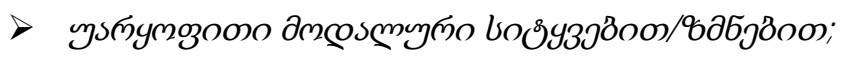

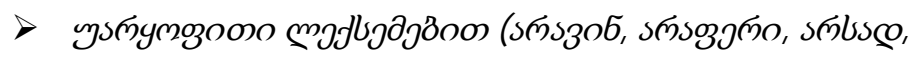

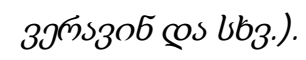

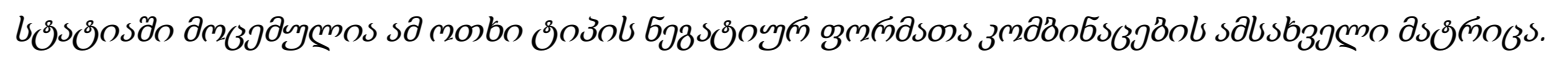

\begin{tabular}{|c|c|c|c|c|c|c|c|c|}
\hline Бэ8s(3os & ১ल(s) & 3э๗(১) & 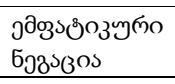 & 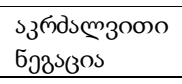 & 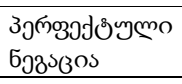 & 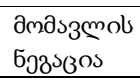 & 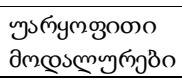 & 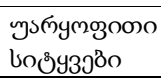 \\
\hline sm(s) & $x$ & Adj./S & Adj./S & Adj./S & Adj./S & $\mathrm{Adj} / \mathrm{S}$ & Adj.past & $S$ \\
\hline $39^{\prime m(s)}$ & Adj./S & $x$ & $\mathrm{~T} / \mathrm{S}$ & 0 & 0 & 0 & $\mathrm{~T} / \mathrm{S}$ & $S$ \\
\hline 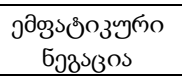 & Adj./S & $\mathrm{T} / \mathrm{S}$ & $\bar{X}$ & 0 & $S$ & $\mathrm{~T} / \mathrm{S}$ & $T / S$ & $S$ \\
\hline 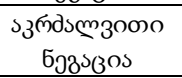 & Adj./S & 0 & 0 & $x$ & 0 & $\mathrm{~S}$ & $S$ & $S$ \\
\hline 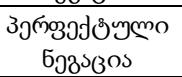 & Adj./S & 0 & $S$ & 0 & $X$ & 0 & $S$ & $S$ \\
\hline 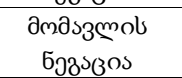 & $\mathrm{Adj} / \mathrm{S}$ & 0 & $\mathrm{~T} / \mathrm{S}$ & $\mathrm{S}$ & 0 & $x$ & $\mathrm{~T} / \mathrm{S}$ & $S$ \\
\hline 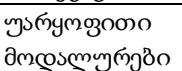 & A dj.past & $\mathrm{T} / \mathrm{S}$ & $T / S$ & $\mathrm{~S}$ & $S$ & $\mathrm{~T} / \mathrm{S}$ & $X$ & $S$ \\
\hline 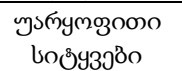 & $\mathrm{S}$ & $\mathrm{S}$ & $\mathrm{S}$ & $\mathrm{S}$ & $S$ & $\mathrm{~S}$ & $S$ & $x$ \\
\hline
\end{tabular}

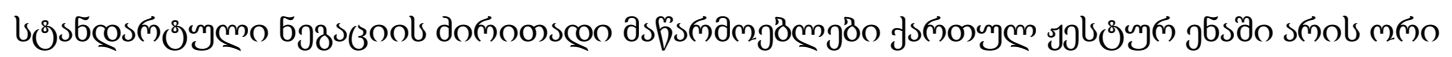

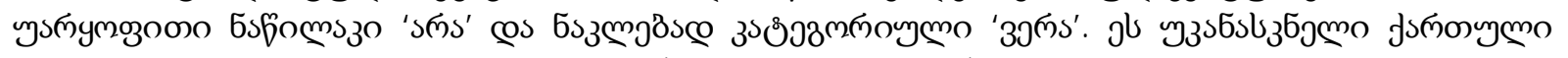

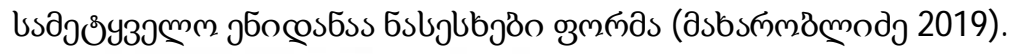

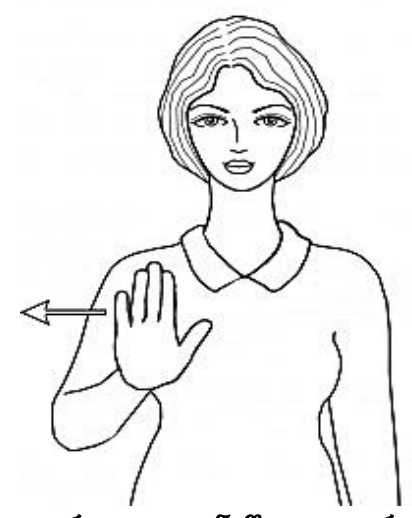

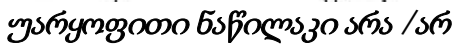

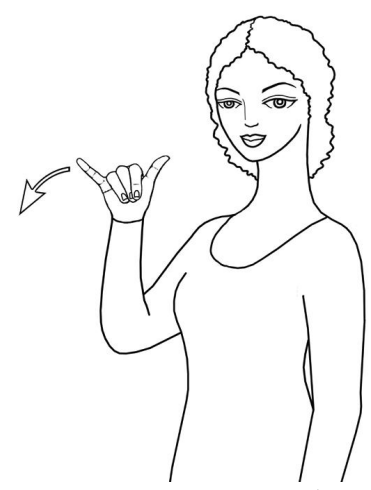

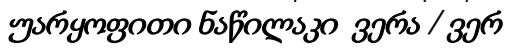




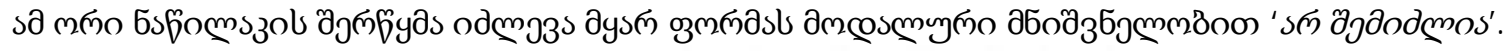

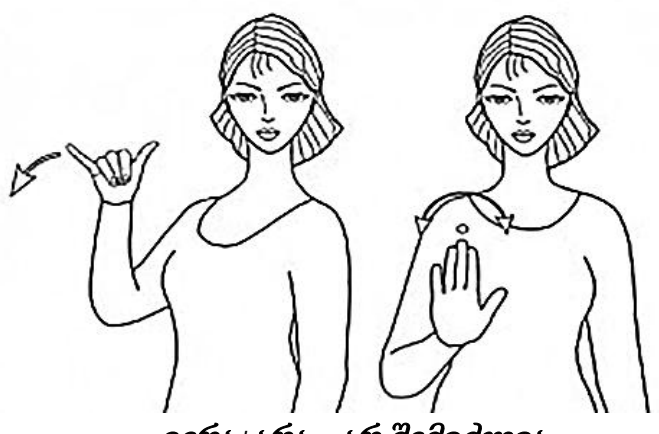

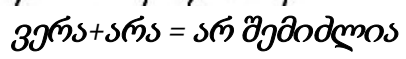

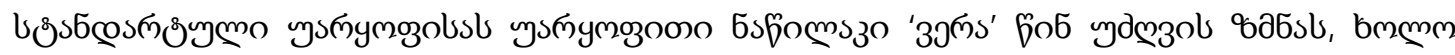

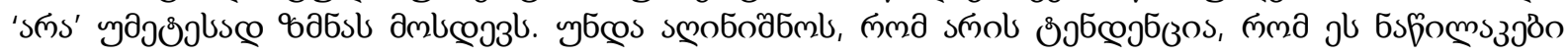

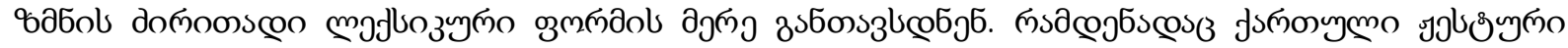

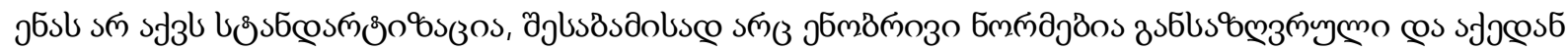

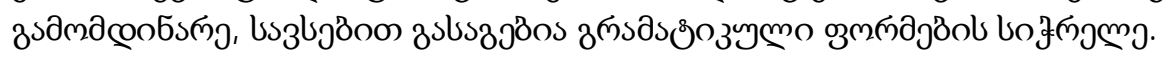

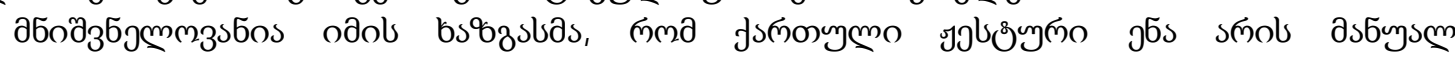

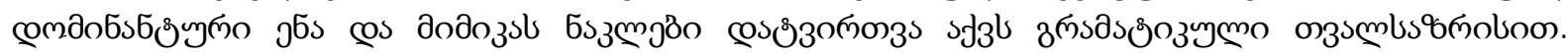

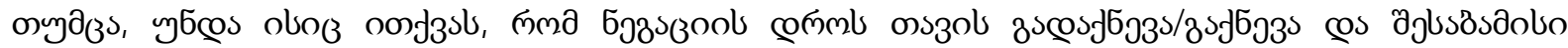

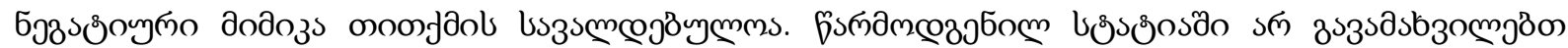

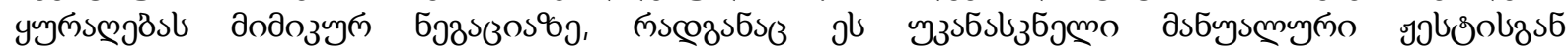

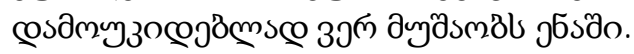
омпдјд̈ою:

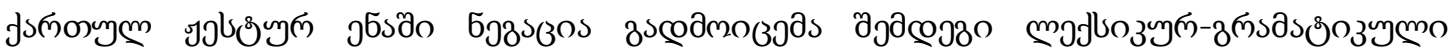

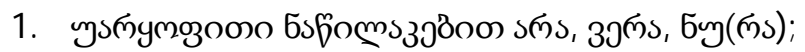

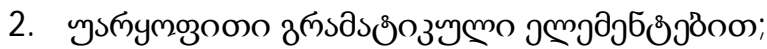

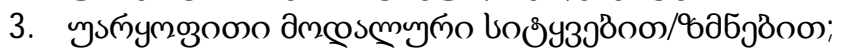

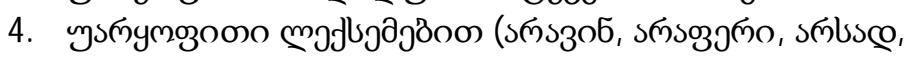
3эलs 306 cos lb3.).

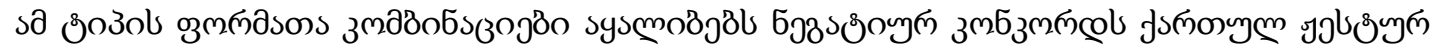

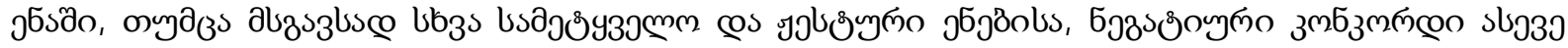

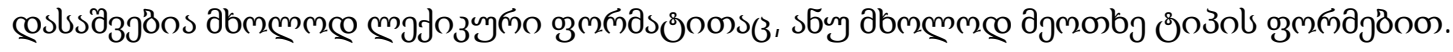

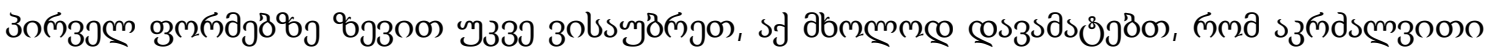

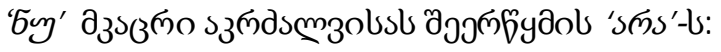

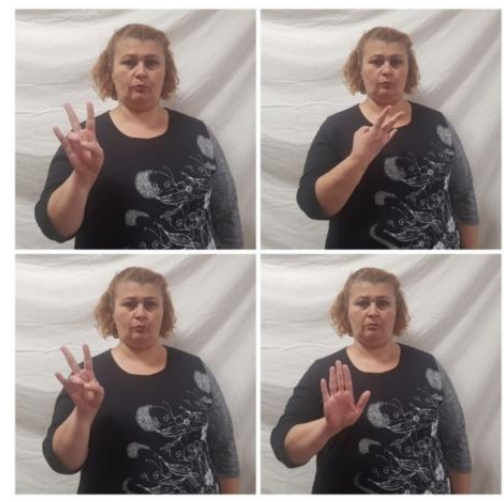

$6 y+s m s$ 


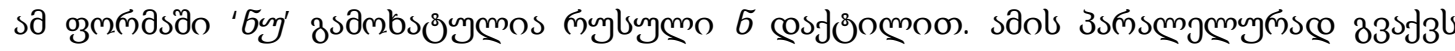

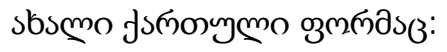

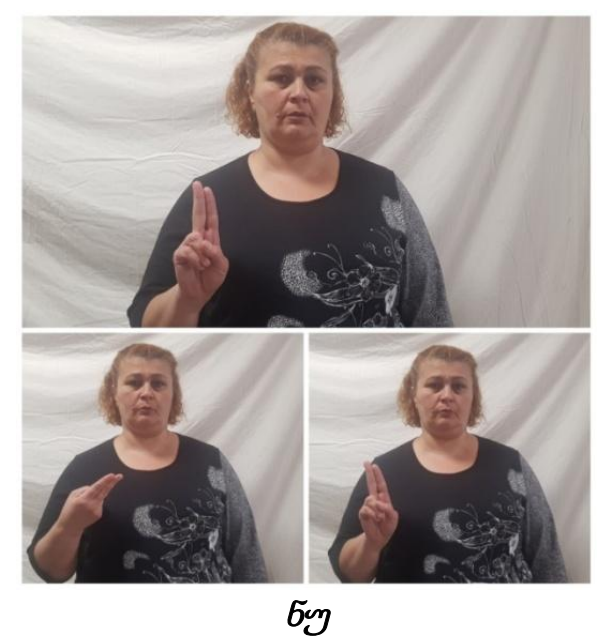

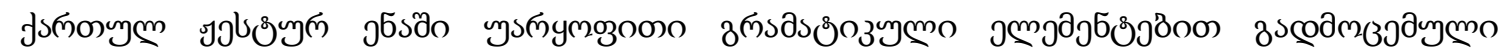

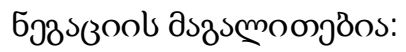

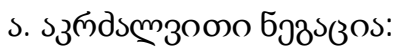

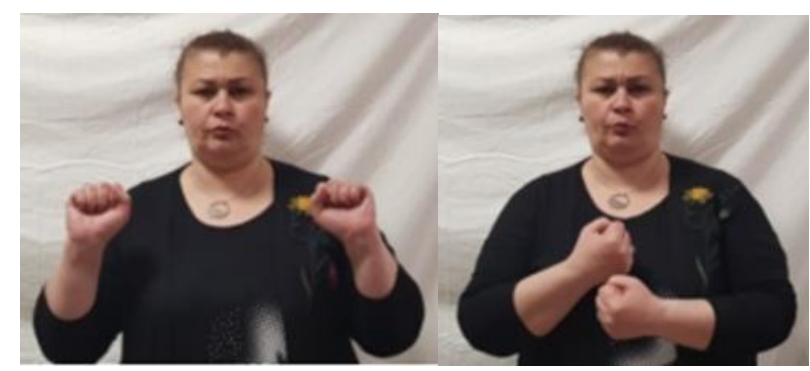

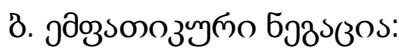

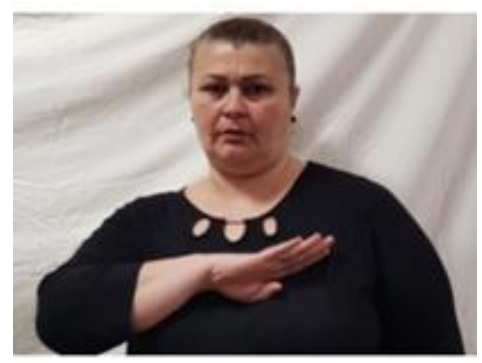

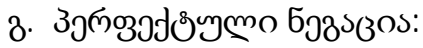




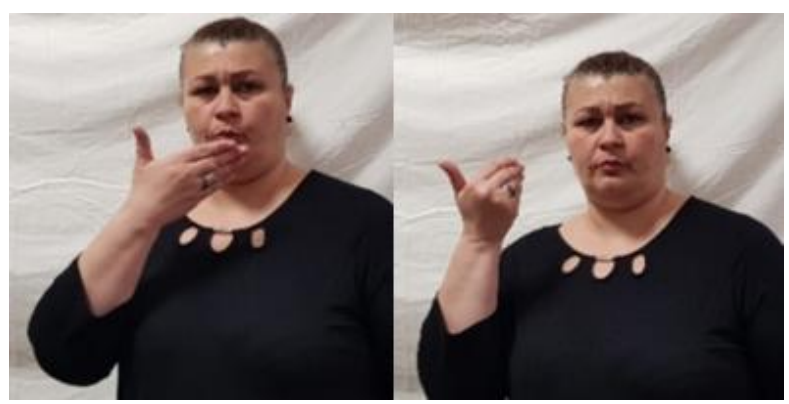

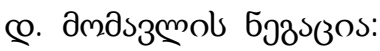

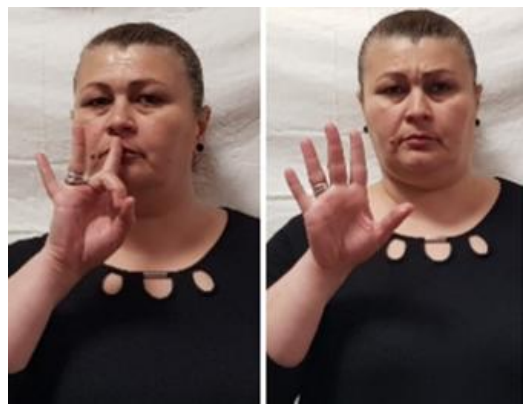

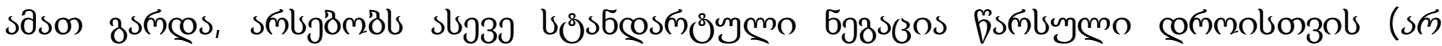

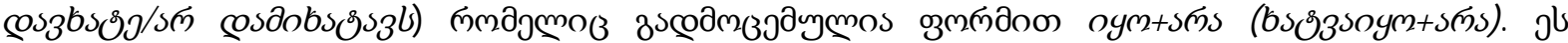

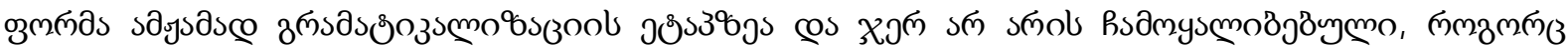

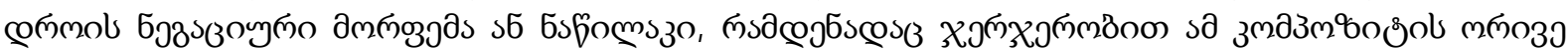

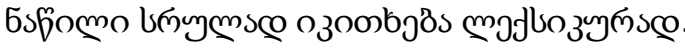

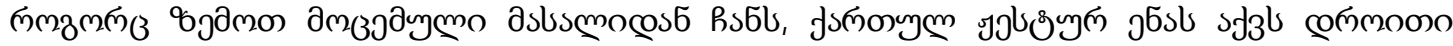

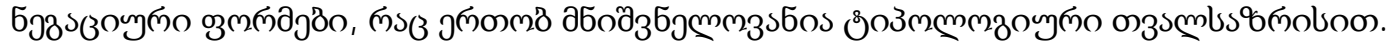

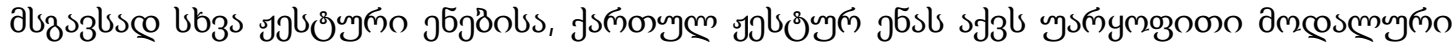

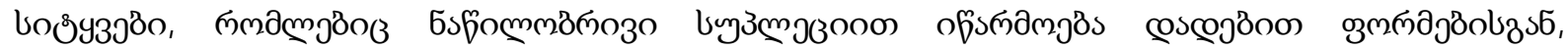
aszsmonsco:

agdodsmos




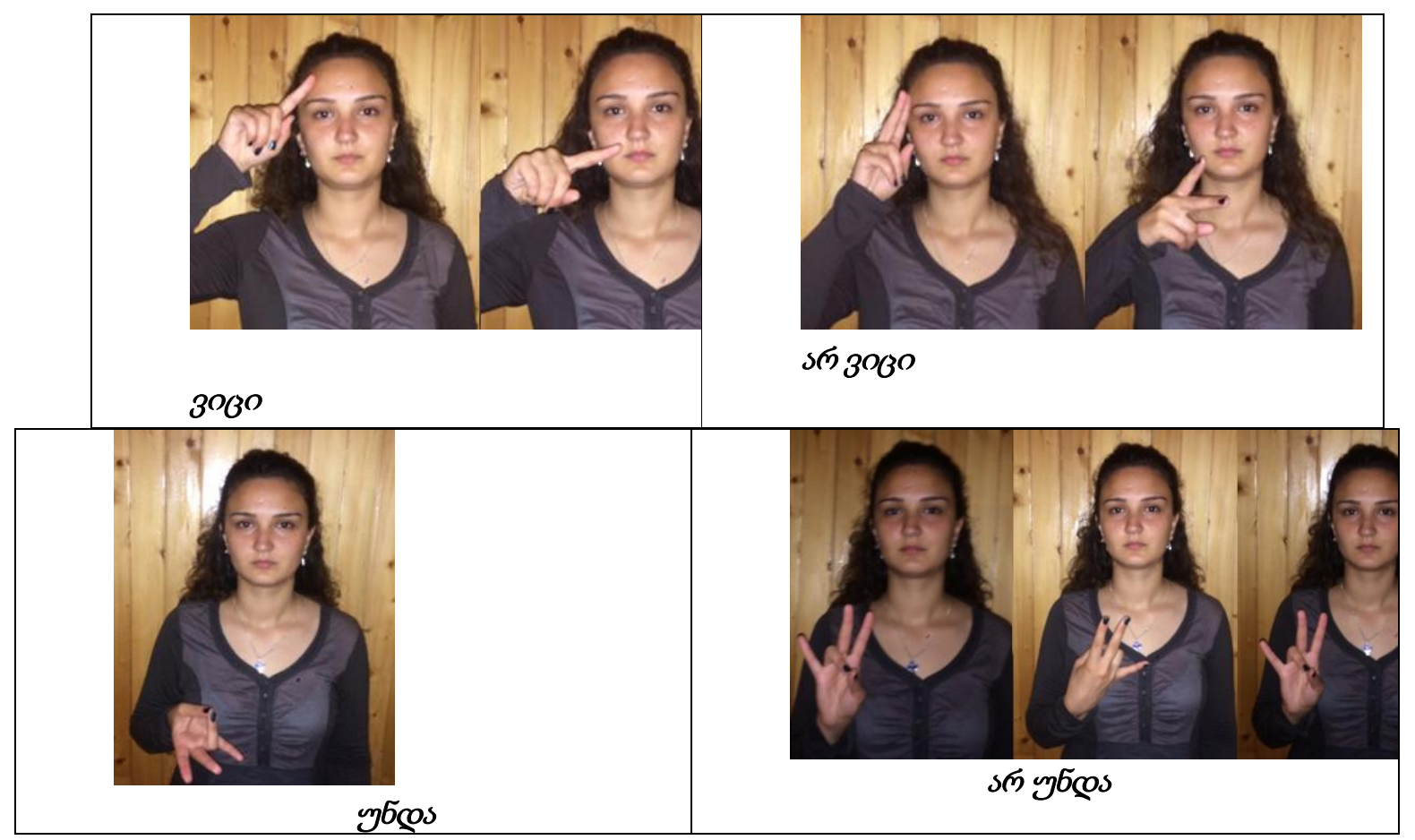

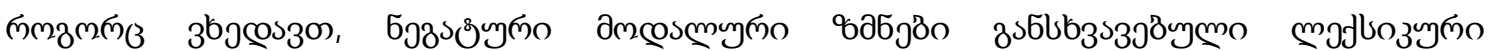

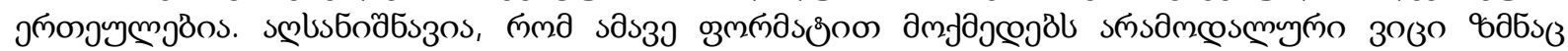

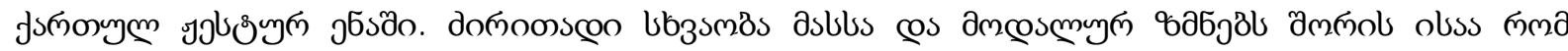

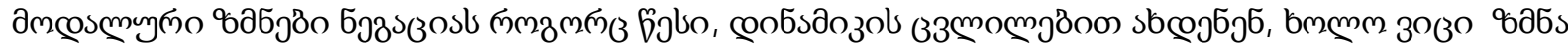

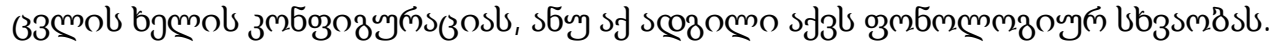

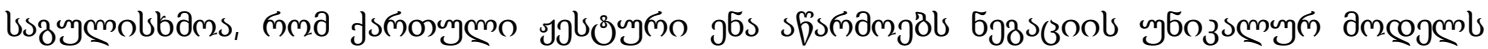

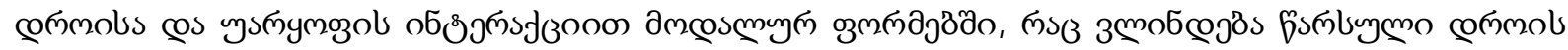

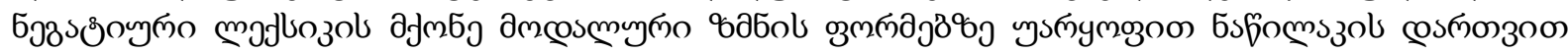

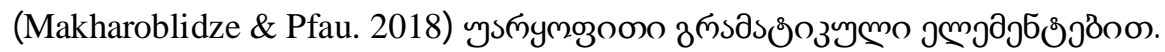

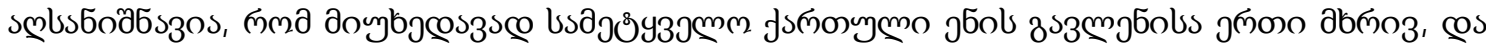

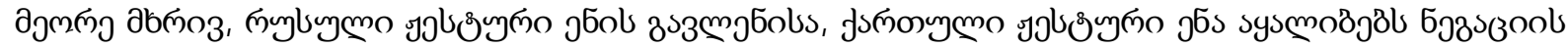

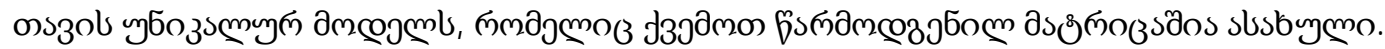

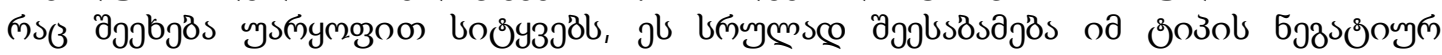

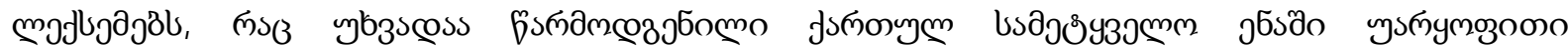

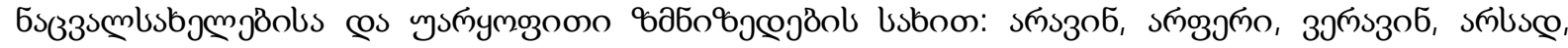

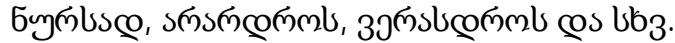

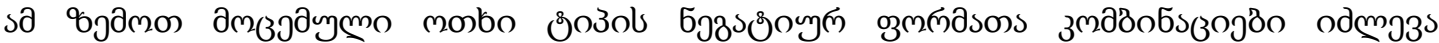

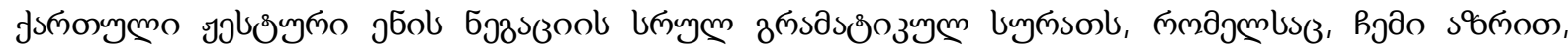

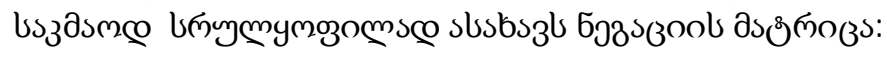

\begin{tabular}{|c|c|c|c|c|c|c|c|c|}
\hline Бэ8s(3)s & $\operatorname{sm}(s)$ & $35^{m(s)}$ & 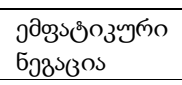 & 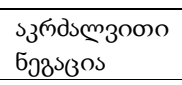 & 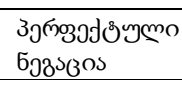 & 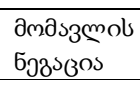 & 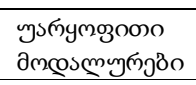 & 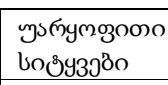 \\
\hline sल(s) & $x$ & Adj./S & $\mathrm{Adj} . / \mathrm{S}$ & Adj./S & Adj./S & $\mathrm{Adj} / \mathrm{S}$ & A dj.past & $S$ \\
\hline 39ळ(s) & Adj./S & $X$ & $\mathrm{~T} / \mathrm{S}$ & 0 & 0 & 0 & $\mathrm{~T} / \mathrm{S}$ & S \\
\hline 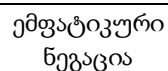 & Adj./S & $\mathrm{T} / \mathrm{S}$ & $x$ & 0 & $\mathrm{~S}$ & $\mathrm{~T} / \mathrm{S}$ & $\mathrm{T} / \mathrm{S}$ & $S$ \\
\hline 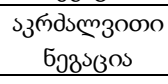 & Adj./S & 0 & 0 & $x$ & 0 & $\mathrm{~S}$ & $\mathrm{~S}$ & S \\
\hline
\end{tabular}




\begin{tabular}{|c|c|c|c|c|c|c|c|c|}
\hline 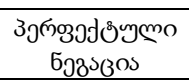 & Adj./S & 0 & $\mathrm{~S}$ & 0 & $x$ & 0 & $S$ & S \\
\hline 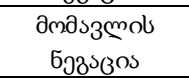 & $\mathrm{Adj} / \mathrm{S}$ & 0 & $\mathrm{~T} / \mathrm{S}$ & $\mathrm{S}$ & 0 & $x$ & $\mathrm{~T} / \mathrm{S}$ & $S$ \\
\hline 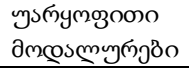 & Adj.past & $\mathrm{T} / \mathrm{S}$ & $\mathrm{T} / \mathrm{S}$ & $S$ & $S$ & $\mathrm{~T} / \mathrm{S}$ & $x$ & $S$ \\
\hline 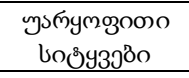 & $S$ & $\mathrm{~S}$ & $S$ & $\mathrm{~S}$ & $S$ & $S$ & $S$ & $x$ \\
\hline
\end{tabular}

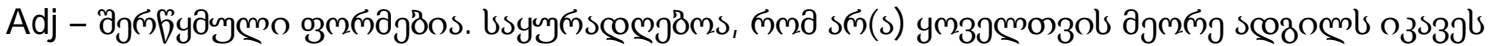

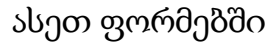

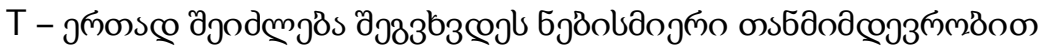

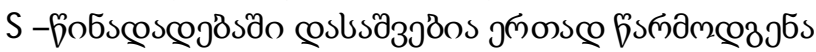

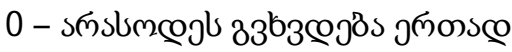

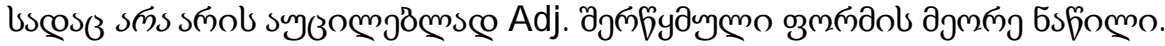

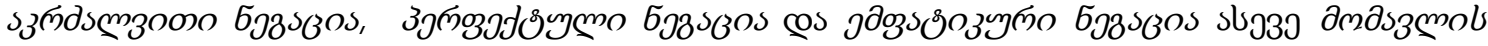

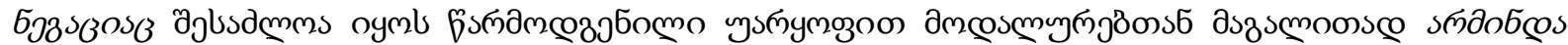

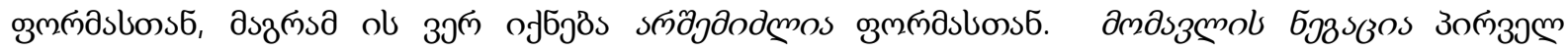

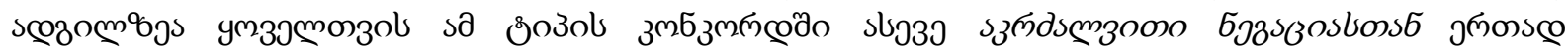

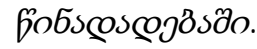

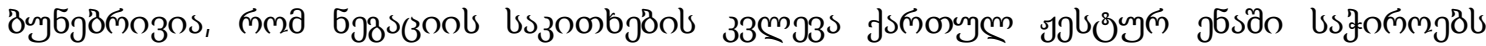

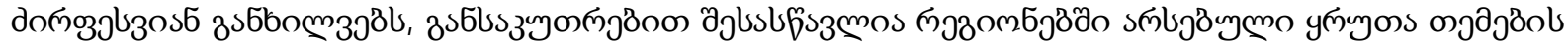

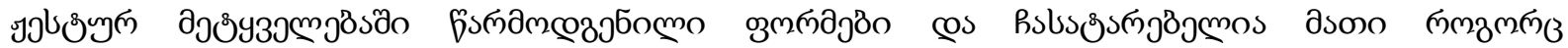

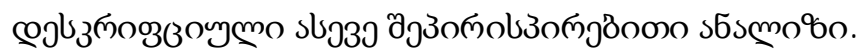

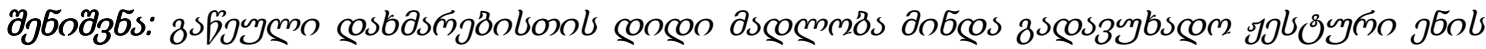

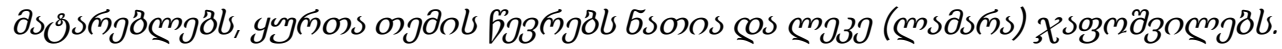

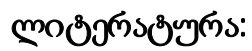

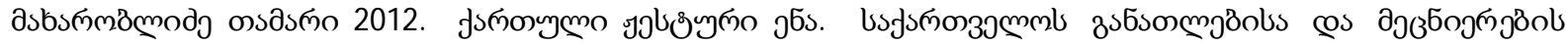

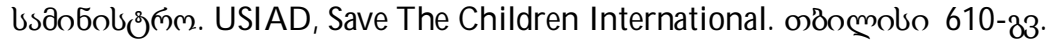

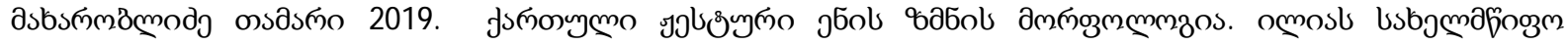

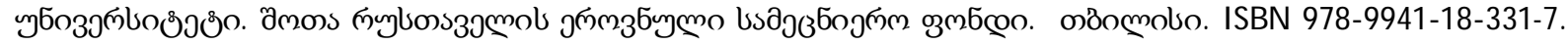
24833 .

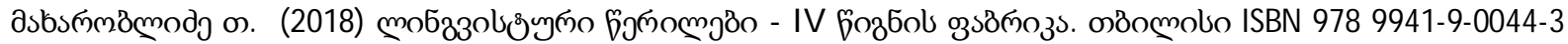
31733 .

Geraci, Carlo. 2005. Negation in LIS (Italian Sign Language). In Leah Bateman \& Cherlon Ussery (eds.), Proceedings of the North East Linguistic Society (NELS 35), 217-229. Amherst, MA: GLSA.

Johnston, Trevor. 2018. A corpus-based study of the role of headshaking in negation in Auslan (Australian Sign Language): Implications for signed language typology. Linguistic Typology 22(2), 185-231.

Makharoblidze, Tamar. 2012. Kartuli jest'uri ena [Georgian Sign Language]. Tbilisi: Ministry of Education and Science, USAID, Save the Children International.

Makharoblidze, Tamar. 2015a. Georgian Sign Language dictionary. Tbilisi: Ilia State University, Shota Rustaveli National Scientific Foundation.

Makharoblidze, Tamar. 2015b. Indirect object markers in Georgian Sign Language. Sign Language \& Linguistics 18(2). 238-250.

Makharoblidze, Tamar. 2019. Kartuli jesturi enis zmnis morpologia [Georgian Sign Language verbal morphology]. Tbilisi: Shota Rustaveli National Science Foundation.

Makharoblidze, Tamar \& Roland Pfau. 2018. A negation-tense interaction in Georgian Sign Language. Sign Language \& Linguistics 21(1), 135-149.

Meir, Irit. 2004. Question and negation in Israeli Sign Language. Sign Language \& Linguistics 7(2), 97-124.

Miestamo, Matti. 2005. Standard negation. Berlin: Mouton de Gruyter. 
Oomen, Marloes \& Roland Pfau. 2017. Signing NOT (or not): A typological perspective on standard negation in Sign Language of the Netherlands. Linguistic Typology 21(1). 1-51.

Pfau, Roland. 2015. The grammaticalization of headshakes: From head movement to negative head. In Andrew D.M. Smith, Graeme Trousdale \& Richard Waltereit (eds.), New directions in grammaticalization research, 9-50. Amsterdam: John Benjamins.

Pfau, Roland. 2016. A featural approach to sign language negation. In Pierre Larrivée \& Chungmin Lee (eds.), Negation and polarity. Experimental perspectives, 45-74. Dordrecht: Springer.

Pfau, Roland \& Josep Quer. 2007. On the syntax of negation and modals in Catalan SL and German SL. In Pamela Perniss, Roland Pfau \& Markus Steinbach (eds.), Visible variation: Comparative studies on sign language structure, 129-161. Berlin: Mouton de Gruyter.

Quer, Josep. 2012. Negation. In Roland Pfau, Markus Steinbach \& Bencie Woll (eds.), Sign language. An international handbook, 316-339. Berlin: De Gruyter Mouton.

Shaffer, Barbra. 2002. CAN'T: The negation of modal notions in ASL. Sign Language Studies 3(1), 34-53.

Wood, Sandra K. 1999. Semantic and syntactic aspects of negation in ASL. West Lafayette, IN: Purdue University Master's thesis.

Zeshan, Ulrike. 2004. Hand, head, and face: Negative constructions in sign languages. Linguistic Typology 8, 1-58.

\section{On Negation in GESL}

Makharoblidze Tamar

Ilia State University,Tbilisi

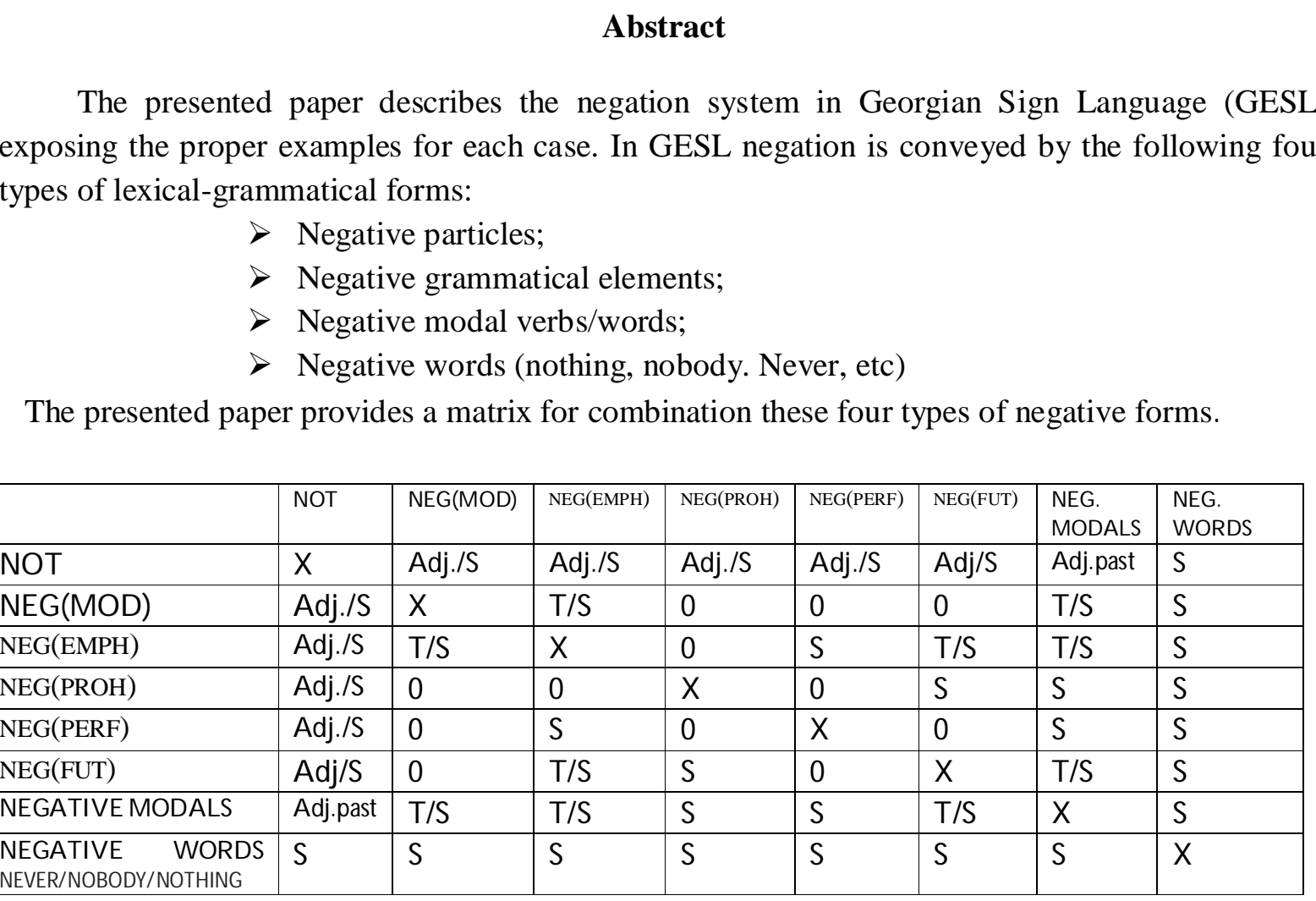

Keywords: Sign languages, Georgian Sign language, negation, negative combinations

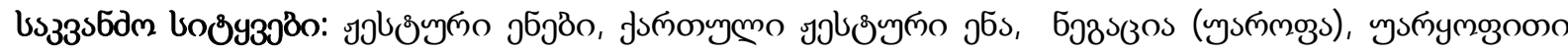
задљобьзојво 